\title{
BMJ Open Understanding clinicians' decisions to offer intravenous thrombolytic treatment to patients with acute ischaemic stroke: a protocol for a discrete choice experiment
}

\author{
Aoife De Brún,, ${ }^{1}$ Darren Flynn, ${ }^{1}$ Kerry Joyce, ${ }^{1}$ Laura Ternent, ${ }^{1}$ Christopher Price,${ }^{2}$ \\ Helen Rodgers, ${ }^{3}$ Gary A Ford, ${ }^{4}$ Emily Lancsar, ${ }^{5}$ Matthew Rudd, ${ }^{2}$ \\ Richard G Thomson ${ }^{1}$
}

To cite: De Brún, A, Flynn D, Joyce $\mathrm{K}$, et al. Understanding clinicians' decisions to offer intravenous thrombolytic treatment to patients with acute ischaemic stroke: a protocol for a discrete choice experiment. BMJ Open 2014;4:e005612.

doi:10.1136/bmjopen-2014005612

- Prepublication history for this paper is available online. To view these files please visit the journal online (http://dx.doi.org/10.1136/ bmjopen-2014-005612)

Received 3 May 2014 Accepted 2 June 2014

CrossMark

For numbered affiliations see end of article.

Correspondence to

Dr Aoife De Brún;

aoife.debrun@ncl.ac.uk

\section{ABSTRACT}

Background: Intravenous thrombolysis is an effective emergency treatment for acute ischaemic stroke for patients meeting specific criteria. Approximately $12 \%$ of eligible patients in England, Wales and Northern Ireland received thrombolysis in the first quarter of 2013, yet as many as $15 \%$ are eligible to receive treatment.

Suboptimal use of thrombolysis may have been largely attributable to structural factors; however, with the widespread implementation of 24/7 hyper acute stroke services, continuing variation is likely to reflect differences in clinical decision-making, in particular the influence of ambiguous areas within the guidelines, licensing criteria and research evidence. Clinicians' perceptions about thrombolysis may now exert a greater influence on treatment rates than structural/service factors. This research seeks to elucidate factors influencing thrombolysis decision-making by using patient vignettes to identify (1) patient-related and clinician-related factors that may help to explain variation in treatment and (2) associated trade-offs in decisionmaking based on the interplay of critical factors.

Methods/analysis: A discrete choice experiment (DCE) will be conducted to better understand how clinicians make decisions about whether or not to offer thrombolysis to patients with acute ischaemic stroke. To inform the design, exploratory work will be undertaken to ensure that (1) all potentially influential factors are considered for inclusion; and (2) to gain insights into the 'grey areas' of patient factors. A fractional factorial design will be used to combine levels of patient factors in vignettes, which will be presented to clinicians to allow estimation of the variable effects on decisions to offer thrombolysis.

Ethics and dissemination: Ethical approval for this study was obtained from the Newcastle University Research Ethics Committee. The results will be disseminated in peer review publications and at national conferences. Findings will be translated into continuing professional development activities and will support implementation of a computerised decision aid for thrombolysis (COMPASS) in acute stroke care.

\section{Strengths and limitations of this study}

- A key strength of this study is that it is the first to use a discrete choice experiment (DCE) to examine clinical decision-making regarding thrombolysis for patients with acute ischaemic stroke.

- The patient vignette approach will offer enhanced clinical realism and will facilitate an examination of the trade-offs that are considered between different patient factors (and levels) when clinicians are making decisions about thrombolytic treatment.

- It is not possible to include in the DCE every possible patient factor that may contribute to variations in decision-making. A structured mixed methods approach must be employed to select patient factors (and levels) that are likely to be contributing most to variation in clinical decision-making about thrombolysis.

- Sampling will be restricted to UK-based clinicians, although the results of the DCE may have relevance to the clinical management of acute stroke in other countries.

\section{INTRODUCTION}

Stroke is a leading cause of death and disability; there are approximately 152000 strokes in the UK every year and between $80 \%$ and $85 \%$ of these are ischaemic strokes. ${ }^{1} \quad$ Intravenous thrombolysis with recombinant tissue plasminogen activator, administered within a maximal time window of 4.5 since onset of symptoms, is the only effective emergency medical treatment available for acute ischaemic stroke, ${ }^{2}{ }^{3}$ and has been recommended by the National Institute for Health and Care Excellence (NICE) since 2007. ${ }^{4}$ Despite the considerable evidence for its efficacy in well-defined patient 
groups, the Sentinel Stroke National Audit Programme (SSNAP) estimated that although approximately 14.3\% of patients were eligible to receive thrombolysis between January and June 2013, only $11.8 \%$ received it. ${ }^{5}$ Furthermore, data from the Stroke Improvement National Audit Programme in 2012 (SINAP) reported wide variation in thrombolytic treatment rates, even between neighbouring services. ${ }^{7}$

Structural factors, such as urgent access to brain CT scanning, initially accounted for much of the variation in thrombolytic treatment rates between centres in the UK; however, with the widespread implementation of 24/7 hyper acute stroke services, continuing variation may reflect differences in clinical decision-making, which is influenced by ambiguous or 'grey' areas within the guidelines, licensing criteria and individual-level interpretation of available research evidence for the efficacy of thrombolysis in the treatment of acute stroke. ${ }^{8}$ As a result, clinicians' decision-making about thrombolysis is likely to be a greater influence on thrombolysis activity and variation in treatment rates than structural service variations. ${ }^{9}$ Factors that might explain variation in clinical decision-making about thrombolysis include the perceived balance between the probability of benefit of treatment and the risk of harm due to intracranial haemorrhage. ${ }^{10}$ However, it remains unclear as to what patient and/or clinician-related factors influence the clinical decision to offer thrombolysis to patients, and especially their relative contributions to observed variation in treatment rates. We aim to identify the factors that contribute to variation in, and influence, clinicians' decision-making about intravenous thrombolysis for patients with acute ischaemic stroke and also to investigate the trade-offs made between different patientrelated factors and levels.

The variation between expert clinicians in clinical decisionmaking about thrombolysis is reflected in an international Delphi study, which sought to establish consensus on the relative contraindications for offering intravenous thrombolysis in acute ischaemic stroke. ${ }^{8}$ Although agreement was reached on 12 of the 18 patient factors, there was a failure to reach consensus on factors such as age, stroke onset time to treatment, recent medical procedures, spontaneous improvement rate and blood pressure treatment. ${ }^{8}$ Licensing criteria currently restricts treatment to patients aged 80 years or below, but most clinicians now treat patients over 80 years on the basis of registry analyses of patients treated in routine practice, recent randomised trial evidence from The Third International Stroke Trial (IST-3) ${ }^{3}$ and increased recognition of the distinction between chronological and physiological age. The European Cooperative Acute Stroke Study III (ECASS III $)^{11}$ trial supported extension of the time window for treatment from 3 to $4.5 \mathrm{~h}$ and the European license time window was extended. ${ }^{12}$ According to the Safe Implementation of Thrombolysis in Stroke-Monitoring (SITS-MOST) study, it is common for patients outside the licensing criteria to be treated, ${ }^{13}$ suggesting that different clinicians are satisfied with different levels of evidence and/ or vary in their decision-making process regarding patient eligibility given the same evidence.

Physician-related and patient-related medical and nonmedical factors can also impact on medical decisionmaking. ${ }^{14-16}$ Influences on the consideration of thrombolysis have been postulated, and include factors such as concern about adverse effects, especially symptomatic intracranial haemorrhage, and uncertainty about effectiveness. ${ }^{9}{ }^{17}$ As others have observed, "managing uncertainty is central to clinical practice and requires the linking of experience and evidence: this places specialists at an advantage" (ref. ${ }^{18}$, p.139). It is important, therefore, to understand how clinician-centred factors such as level of expertise, age, seniority, practice setting and attitude to risk can influence the decision to offer thrombolysis. ${ }^{19}$ The situation is further complicated by the absence of data on outcomes as a function of individual patient characteristics, lack of quality decision support tools ${ }^{20}$ and lack of consensus on relative contraindications. ${ }^{8}$ Additional patient factors, such as prior cognitive function, prior functional ability and comorbidities are also likely to influence decision-making. ${ }^{14}$ Nevertheless, little is known about the relative importance of these medical and nonmedical factors and indeed whether or not other patientrelated medical and non-medical factors, including clinician-related factors, are at play. Furthermore, some of these factors are likely to reflect clinical criteria, whereas others are interpretations of criteria and evidence which is amenable to education/behaviour change or better presentation of evidence for under and overtreatment.

\section{Aims}

The study aims to identify the factors that contribute to variation in, and influence, clinicians' decision-making regarding the use of thrombolysis for treatment of patients with acute ischaemic stroke. Specifically, the objectives of the project are:

1. To determine which patient factors influence clinical decision-making about the offer of thrombolysis;

2. To identify and quantify the trade-offs between patient factors/levels that clinicians make regarding the decision to offer thrombolysis and variation in this decision;

3. To determine which clinician-related factors influence clinical decision-making about the offer of thrombolysis (eg, clinician experience, clinical specialty and attitude to risk);

4. To influence clinicians' behaviour by translating learning into continuing professional development (CPD) activity, national clinical guidelines, supporting implementation of an existing thrombolysis decision support tool and informing clinical audit and evaluation programmes (SSNAP).

\section{METHODS AND ANALYSIS}

To examine the factors that influence clinicians' decision-making when considering thrombolysis, it is 
important to understand their thought processes when they are making these decisions in a way that reflects decision-making in practice. An online discrete choice experiment (DCE) utilising hypothetical patient vignettes will be conducted to better understand how clinicians make decisions about whether or not to offer thrombolysis, and to capture the underpinning tradeoffs made in decision-making. To inform the final design of the patient vignettes and clinician-related factors for inclusion in the DCE, exploratory work will be undertaken to ensure, insofar as is possible, that all potentially relevant factors are identified for inclusion in the study. This exploratory work will adopt a mixed methods approach to facilitate optimal design of the DCE. Figure 1 presents an overview of the processes involved.

\section{Rationale for a DCE to examine clinical decision-making about thrombolysis}

The DCE approach offers a means to examine the nuances of decision-making, by providing insights into the (sometimes implicit) trade-offs made between different factors and levels, which are not easily captured through other more traditional research methods. Based on the tenets of random utility theory, ${ }^{21}$ a DCE is designed to elicit participants' preferences and the relative importance of different factors/levels within a decision-making model. ${ }^{22}$ DCEs assume that there is more than one factor influencing decision-making and that all choices involve trade-offs. ${ }^{23}$ Ryan et al (ref. 23, p.15) observe that, since "researchers cannot get inside the heads of these individuals (clinicians) and observe all factors affecting preferences," a DCE offers a means to explore and quantify the implicit trade-offs made between multiple competing factors within a decisionmaking scenario. This method can be used to systematically assess preferences within any given decision-making scenario and thereby can be used to access the underpinning cognitions of clinicians which lead to a decision about the offer of thrombolysis. DCEs are widely used in the health sector ${ }^{24}$ including decision-making in areas such as clinical recommendation of contraceptive type ${ }^{25}$ and investigating participant preferences for preventative asthma medication. ${ }^{26}$ While DCEs have been undertaken in the field of stroke medicine (eg, to explore patient preferences for early rehabilitation management) ${ }^{27}$ none have examined the factors that influence clinicians' decisions to offer thrombolysis to acute ischaemic stroke patients.

Clinicians involved in thrombolysis decision-making will be presented with hypothetical patient vignettes which vary in terms of their characteristics (patient factors, eg, age) and the magnitude of these characteristics (levels, eg, patient age 75 or 85 years) and asked to state whether they would offer thrombolysis. ${ }^{22}$ The relative importance of different patient factors (and levels) to the decision can then be quantified, and the estimated choice model then used to predict the probability of offering thrombolysis given certain characteristics of the patient. ${ }^{22}{ }^{28}$ This will enable insight into the patient factors and levels which influence the decision to offer thrombolysis. An example of a draft patient vignette is provided in figure 2. The DCE will also examine observed variation in decision-making by clinician factors such as age, experience, clinical background and attitude to risk (using a six-item version of the Jackson Personality Inventory Risk-taking subscale) ${ }^{19} 29$

\section{Exploratory work to establish factors and levels that potentially influence clinical decision-making about thrombolysis}

In-depth exploratory work, usually involving qualitative methods and literature review, is an important first stage in any DCE to establish the factors (characteristics of the decision-making model) and levels (magnitude of those characteristics) for inclusion. ${ }^{30}$ This research will draw on previously completed work by the project team for the Developing and Assessing Services for Hyperacute (DASH) Stroke NIHR-funded programme grant for applied research. ${ }^{31}$ This research explored optimal organisation of prehospital and acute stroke care and aimed to improve outcomes for stroke patients by building on the evidence base for future health service interventions. The programme examined barriers to acute stroke patient admission, the views of clinicians, the public, stroke patients and their relatives/carers about the risks and benefits of thrombolysis and how to engage patients and their families in decisionmaking. ${ }^{32}{ }^{33}$ In the current study, we will re-examine qualitative interview data on the views and experiences of stroke clinicians about thrombolysis decision-making, as well as ethnographic data collected through participant observation and data from the usability testing of a COMPuterised decision Aid for Stroke thrombolySis (COMPASS) to ensure that potentially relevant patient and clinician factors in thrombolysis decision-making are considered. $^{32} 33$ In addition, semistructured one-on-one interviews will be conducted with up to 10 additional stroke clinicians who were not involved with the DASH study but are based in centres outside the North East of England. Interviews will be audiorecorded and transcribed verbatim. The sampling strategy will be purposive, to include clinicians from the local cardiovascular network and beyond, and will seek to include clinicians from hospitals in the upper and lower quartiles of thrombolysis rates, based on figures from national audit reports. $^{5-7}$

Participants will be asked to reflect on their clinical experience where thrombolysis was considered; in particular, they will be asked to talk through the typical decision-making process and then asked to recall grey, borderline and/or difficult cases, including trade-offs made and perceived influential factors on their decision. Additional prompts will include: influential factors beyond guidelines and licensing criteria; experiences of adverse events; communicating risks and benefits of 
Figure 1 Overview of the discrete choice experiment development process.

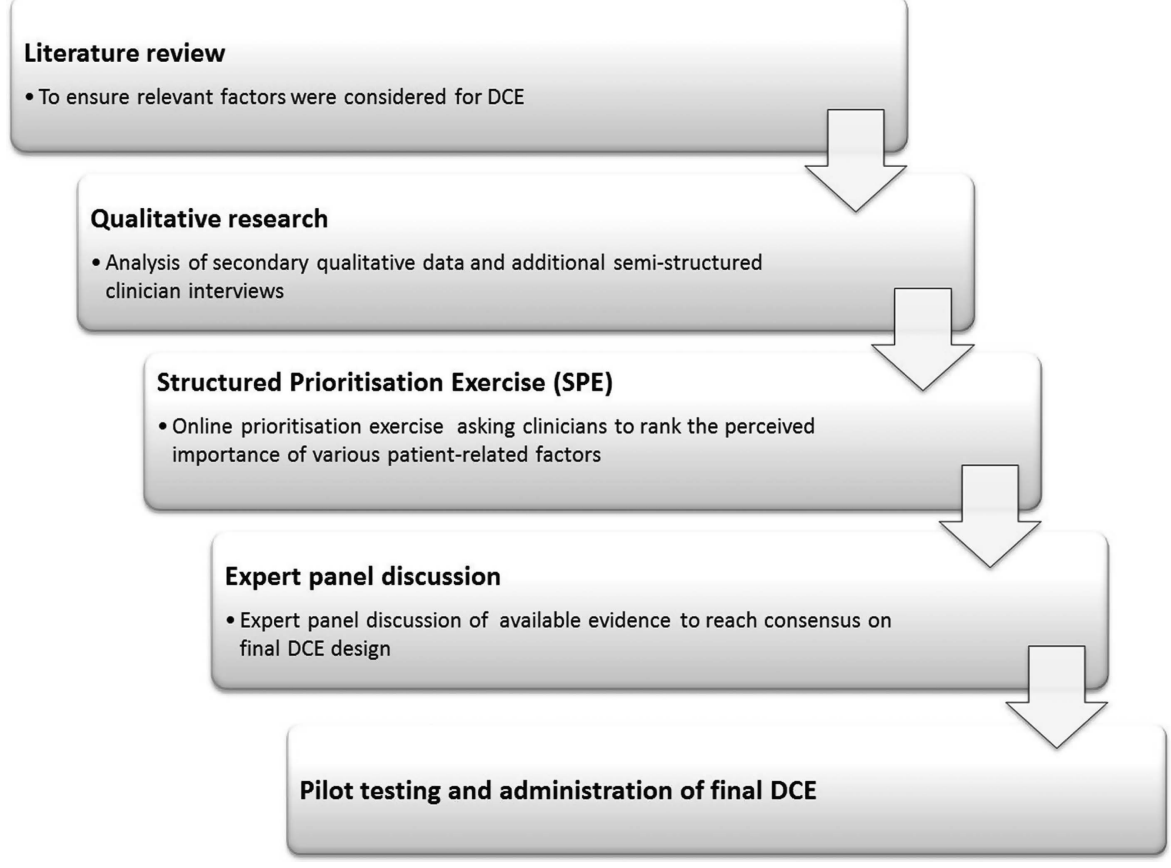

thrombolysis; attitude to risk; colleagues' views and experiences; and approaches and norms within their practice setting. Anonymised transcripts will be imported into QSR International's NVivo 9 to facilitate the coding and analysis of data. ${ }^{34}$ The resulting data set will be coded thematically and analysed using a framework approach to establish the range of factors (and levels) that could be included in the DCE. ${ }^{35}$ In short, these interviews will be used to identify any additional patientrelated and clinician-related factors that might influence decision-making that are not accounted for in the existing qualitative data set from the DASH research programme or in the published literature.
The exploratory phase will enable the generation of a comprehensive list of patient-related and clinicianrelated factors that potentially influence decision-making about whether or not to offer thrombolysis to patients with acute ischaemic stroke. This list will then be used to inform the design of a structured prioritisation exercise (SPE). The SPE will include patient-related factors (and levels or magnitudes of these factors) that will assist us in selecting the most influential factors on clinical decision-making, and also facilitate the elucidation of where 'grey' areas (ie, areas of uncertainty) exist in decision-making about thrombolysis. Questions within the SPE will be designed to assist in determining (and
Figure 2 Example of a draft vignette.
A frail 81 year old woman is admitted to hospital with an ischaemic stroke. She has no prior history of stroke and has been living independently. Clinical assessment of stroke severity indicated an NIHSS score of 18 . On examination, her systolic blood pressure was $165 \mathrm{mmHg}$ and further investigations revealed blood glucose levels of $16.6 \mathrm{mmol} / \mathrm{L}$ and an INR of 1.7. It is 1.5 hours since the onset of her stroke symptoms.

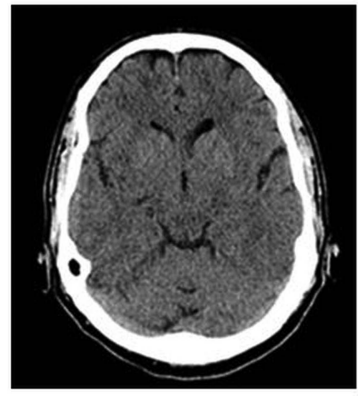

This CT scan was conducted at 100 minutes and shows no evidence of intracranial haemorrhage.

Would you offer thrombolysis to this patient?

YES $\square$

NO $\square$ 
operationalising) the relative importance of patientrelated factors (and levels) for decision-making about thrombolysis, as well as providing insight into the nature of 'grey' areas that exist for each patient factor. The first phase of the SPE will be hosted online and will be circulated to up to 30 stroke clinicians responsible for thrombolysis decision-making. The results of the SPE will be examined using frequency analysis to inform discussions with an expert panel in the second phase (consisting of three stroke physicians, a trainee stroke physician, two stroke patient representatives, two health psychologists, two health economists and a specialist in epidemiology, public health and decision-making) to determine which patient-related factors and levels will be included in the patient vignettes. This panel will also deliberate on the most relevant clinical information to be presented in the patient vignettes to ensure that they are representative of a real-world patient assessment and provide sufficient information to allow clinicians to reach a decision about whether or not to offer thrombolysis to patients with acute ischaemic stroke.

\section{Experimental design of DCE}

A fractional factorial design will be used to combine patient-related factors and levels to construct hypothetical patient vignettes. The number of factors and levels will be identified from initial exploratory work. The narrative format of vignettes, rather than the traditional tabular lists of factors and levels, will be used to increase participant engagement by providing clinical face validity. ${ }^{36}$ After presentation of each vignette, participants will be asked whether they would offer thrombolytic treatment or not (see figure 2 for an example). To further enhance clinical validity, all scenarios will be discussed with stroke clinicians in the project management group to ensure that they are plausible and clinically appropriate and provide an adequate range and variety of cases. To further enhance external validity (and allow comparison of model predictions to actual cases), a subsection of vignettes will be based, as closely as possible, on real cases identified through SITS-UK (thrombolysed cases only), ${ }^{37}$ and a database of anonymised cases (treated and nontreated) collected as part of a local audit.

Vignettes will also include CT imaging scans/descriptions of CT scans to make the decision-making process as realistic as possible and to mirror actual clinical situations. However, to remove subjectivity around the interpretation of CT imaging, a brief description of the accompanying CT scan findings will also be provided. Importantly, the experimental design will allow us to include a number of vignettes that show variation in patient factors that are likely to be most sensitive to differing decisions, that is, those difficult 'grey zone' cases. The vignettes will be reduced to a manageable number using experimental design techniques (fractional factorial design) and incorporated into an online survey. Within the survey, the case vignettes will be followed by a series of questions on clinician factors to be used in the estimation of choice models as covariates such as clinician age, grade/seniority, experience with thrombolysis and personality type/risk aversion (using the Jackson Personality Inventory Risk-taking subscale). ${ }^{19} 29$

The DCE will be programmed into an online survey to enhance the ease and speed of survey dissemination and data collection. The survey will be pretested, with a convenience sample of up to eight clinicians, to establish the clinical validity of the vignettes, examine participants' understanding and check how long the survey takes to complete. Pretesting will include a think-aloud approach, ${ }^{38}$ using a subset of vignettes, to facilitate adaptation of the vignettes and survey instrument. This will address practical issues, such as length of the survey to avoid response fatigue, ${ }^{21} 28$ optimal presentation of choice sets (vignettes) and views on how to make the online survey easier to use. ${ }^{28}$ The vignettes will be designed to mimic as closely as possible the decision of interest. This will be in the form of a binary response (decision to offer thrombolysis or not) as this reflects routine clinical practice whereby clinicians are faced with one patient at a time and must decide whether or not to offer thrombolysis. Clinicians tend to be familiar, and are likely to be more comfortable, with vignettes as they are regularly used in training and CPD. Consequently, participating clinicians may treat the DCE as a test and thus behave differently to how they would in the real world. As such, the information sheet preceding the DCE survey will clearly communicate that all responses will be confidential and individuals will not be judged on their responses against guidelines/licensing criteria for thrombolysis.

\section{Participant sampling and recruitment}

Optimal sample size requirements for DCEs depend on knowledge of the true choice probabilities, which are not known prior to undertaking the research. ${ }^{39}$ The final sample size required will also depend on the ultimate design of the DCE, that is, the number of patient factors included and the number of levels of each factor, including consideration of the response rate from the target population. Previous DCE studies have shown that robust choice models can be estimated from sample sizes between 50 and 100 respondents. ${ }^{26} 39$

Clinicians will be recruited via the British Association of Stroke Physicians (BASP) ${ }^{40}$ with reminders issued at 2 and 4 months to maximise the response rate. An invitation to participate, together with an information sheet, will be distributed to members of BASP with a link to the online DCE survey. In some cases, emergency department physicians and neurologists may have independent responsibility for decision-making about thrombolysis. Therefore, to take account of the diversity in service configuration, an invitation to participate will also be circulated nationally via stroke research network regional leads and via the Society for Acute Medicine. Eligibility for participation in the survey will be defined as the potential participant having independent 
responsibility for decision-making about thrombolysis. Physicians involved only in discussions about the offer of thrombolysis will be excluded. To ensure that these criteria are adhered to, a question about the respondent's role in decision-making about thrombolysis will be included in the introductory screening questions.

\section{Analytical plan}

The data will be modelled in a random utility theory framework using limited dependent variable multiple logistic (hierarchical) regression modelling, in particular using binary logit models and extensions of such models. The decision to offer thrombolysis will be modelled as a function of the patient factors/levels described in the vignettes and the characteristics of the decisionmakers (clinician factors). This will allow investigation of which patient factors (and levels) drive or inhibit the clinical decision to offer thrombolysis, as well as which characteristics of the decision-makers (eg, age, experience of offering thrombolysis, attitudes to risk) influence the clinical decision to offer thrombolysis (observed heterogeneity). Interaction of such clinician characteristics with patient factors will allow exploration of how the importance of patient factors/levels may vary by clinician characteristic (eg, specialty, level of expertise, degree of experience, etc). Unobserved heterogeneity will be explored using random coefficients and scale-adjusted latent class modelling. Using the preferred model (based on goodness of fit criteria), ${ }^{23}$ marginal rates of substitution will be calculated to quantify the rate at which clinician respondents are prepared to trade off one patient factor for another, which also allows investigation of the relative importance, or ranking, of the characteristics of patients to the decision to offer thrombolysis. ${ }^{30}{ }^{41}$ Predicted probability analysis will not only investigate the probability of offering thrombolysis using clinically relevant values on the levels of factors but will also be used in the exploration of external validity.

\section{ETHICS AND DISSEMINATION}

Interview participants will be informed that any personal details will be removed from interview transcripts and will not be disclosed in any reports or publications. As the DCE will be conducted via an online survey, it will not be possible to collect signed consent forms from participants. Instead, the opening page of the online survey will clearly state that participation is voluntary and there is a right to withdraw participation at any stage, without reason. Participants will be advised that answers will be confidential. They will then be given a brief description of the study (with contact details of the lead researcher should they have any questions) and asked to indicate their consent to participate by ticking a box and continuing through the online survey.

The DCE will be used to establish:(1) which patient factors/levels described in the vignettes are taken account of by clinicians when deciding to offer thrombolysis; (2) which of these factors act as drivers to, or have a positive impact on, the decision to offer thrombolysis, and which act as inhibitors, or reduce the likelihood of offering thrombolysis and (3) how these influence the appropriate use of thrombolysis. The influence of characteristics of the clinical decision-makers on such decisions will also be explored.

These data will be used to increase appropriate use of thrombolysis for patient benefit by influencing clinicians' knowledge, skills and behaviour via four routes.

- The findings will be incorporated into postgraduate training and continuing professional development activities relevant to thrombolysis in acute stroke care. This will be undertaken through the development, revision and sharing of training materials with the National Health Service (NHS) Improving Quality Programme, so that training for stroke physicians and teams can incorporate the study findings. ${ }^{40} \quad 42-44$ Results will also be presented at the UK Stroke Forum and British Association of Stroke Physicians annual trainees' event.

- The findings will also be used to support implementation of decision support by building on the outputs of the previous DASH work, which included the development of a computerised decision aid for thrombolysis -COMPASS. ${ }^{45}$ The results will enable the development of tutorial information to be embedded within COMPASS, with the aim of optimising its use as a clinical training aid. Information on factors known to drive or inhibit clinicians' decisions to offer thrombolysis will be included within the tutorial to optimise use. Providing additional information and decision support to clinicians will lead to better support for risk communication, consent and decision-making about thrombolysis with patients/relatives.

- The study outputs will be linked to national clinical audit (SSNAP). Through this mechanism, it is possible that the study findings can be used to determine whether additional data items (eg, use of decision support tools such as COMPASS) should be routinely collected about thrombolysis at a national level, and they will also help in the interpretation of the findings of the national audit.

- Finally, the results will be disseminated as national conference presentations, peer reviewed papers and reports to the Stroke Research Network, the Stroke Association and the Intercollegiate Stroke Working Party to be considered for inclusion in National Clinical Guidelines.

\section{Author affiliations}

${ }^{1}$ Institute of Health \& Society, Newcastle University, Newcastle upon Tyne, UK ${ }^{2}$ Northumbria Healthcare NHS Foundation Trust, Ashington, UK

${ }^{3}$ Institute for Ageing and Health (Stroke Research Group), Newcastle University, Newcastle upon Tyne, UK

${ }^{4}$ Oxford University Hospitals NHS Trust, Oxford, UK

${ }^{5}$ Centre for Health Economics, Monash University, Clayton, Melbourne, Victoria, Australia 
Acknowledgements The authors would like to express their thanks to John Teah and Stephen Simpson (public representatives from The Stroke Association) for their valuable and continuing contribution to the research.

Contributors RGT, GAF, DF, KJ, LT, EL, HR and CP were responsible for the conceptual design of the study. All authors were involved with revisions to the design of the DCE and drafting of this manuscript.

Funding This project is funded by the National Institute for Health Research Health Services and Delivery Research Programme (project number: 12/5001/45). The views and opinions expressed therein are those of the authors and do not necessarily reflect those of the HS\&DR Programme, NIHR, NHS or the Department of Health.

Competing interests DF, GAF, HR, CP and RGT have been involved in marketing activity for COMPASS, which may be made available for a cost payable to purchase the decision aid to cover the costs of technical maintenance and updating of the information content (predictive equations and user interface) in accordance with user feedback and availability of new data on the effectiveness of thrombolysis. GAF's previous institution has received research grants from Boehringer Ingelheim (manufacturer of Alteplase), and honoraria from Lundbeck for stroke-related activities. GAF has also received personal remuneration for educational and advisory work from Boehringer Ingelheim and Lundbeck. Also, GAF is supported by an NIHR Senior Investigator award.

Ethics approval Favourable ethical opinion for the study was obtained from the Newcastle University Research Ethics Committee.

Provenance and peer review Not commissioned; internally peer reviewed.

Open Access This is an Open Access article distributed in accordance with the Creative Commons Attribution Non Commercial (CC BY-NC 4.0) license, which permits others to distribute, remix, adapt, build upon this work noncommercially, and license their derivative works on different terms, provided the original work is properly cited and the use is non-commercial. See: http:// creativecommons.org/licenses/by-nc/4.0/

\section{REFERENCES}

1. Townsend N, Wickramasinghe K, Bhatnagar P, et al. Coronary heart disease statistics 2012 edition. London: British Heart Foundation, 2012

2. Wardlaw JM, Murray V, Berge E, et al. Recombinant tissue plasminogen activator for acute ischaemic stroke: an updated systematic review and meta-analysis. Lancet 2012;379:2364-72.

3. Sandercock P, Wardlaw JM, Lindley RI, et al.; IST-3 Collaborative Group. The benefits and harms of intravenous thrombolysis with recombinant tissue plasminogen activator within $6 \mathrm{~h}$ of acute ischaemic stroke (The third international stroke trial [IST-3]): a randomised controlled trial. Lancet 2012;379:2352-63.

4. National Institute for Health and Clinical Excellence. Alteplase for the treatment of acute ischaemic stroke, NICE technology appraisal guidance 122. London: National Institute for Health and Clinical Excellence; 2007

5. Royal College of Physicians. Clinical Effectiveness and Evaluation Unit on behalf of the Intercollegiate Stroke Working Party. Clinical audit second pilot public report: National results based on stroke patients admitted to and/or discharged from hospital between AprilJune 2013. http://www.rcplondon.ac.uk/sites/default/files/ssnap_ public_report_2_-_national_level_results_april_-_june_2013_with appendices.pdf

6. Royal College of Physicians. Clinical Effectiveness and Evaluation Unit on behalf of the Intercollegiate Stroke Working Party. Clinical audit first pilot public report: National results based on stroke patients admitted to hospital between January-March 2013. http:// www.rcplondon.ac.uk/sites/default/files/ssnap first pilot national report_january_-_march_2013_admissions_with_appendices_pdf

7. Royal College of Physicians. SINAP_latest results; JanuaryDecember 2012. http://www.rcplondon.ac.uk/resources/ sinap-latest-results

8. Dirks M, Niessen LW, Koudstaal PJ, et al. Intravenous thrombolysis in acute ischaemic stroke: from trial exclusion criteria to clinical contraindications. An international Delphi study. J Neurol Neurosurg Psychiatry 2007;78:685-9.

9. Kwan J, Hand P, Sandercock P. A systematic review of barriers to delivery of thrombolysis for acute stroke. Age Ageing 2004;33:116-21.
10. Ciccone A. Consent to thrombolysis in acute ischaemic stroke: from trial to practice. Lancet Neurol 2003;2:375-8.

11. Bluhmki E, Chamorro Á, Dávalos A, et al. Stroke treatment with alteplase given 3.0-4.5 h after onset of acute ischaemic stroke (ECASS III): additional outcomes and subgroup analysis of a randomised controlled trial. Lancet Neurol 2009;

8:1095-102.

12. Ingelheim B. Boehringer Ingelheim gains approval of extended 4.5 hour time-window for Actilyse $\AA$ in acute ischaemic stroke for majority of EU countries. 2011. http://www.boehringer-ingelheim.com/news/ news_releases/press_releases/2011/04_november_2011_alteplase. html

13. Toni D, Lorenzano S, Puca E, et al. The SITS-MOST registry Neurol Sci 2006;27:s260-2.

14. Flynn $D$, van Schaik $P$, van Wersch $A$, et al. Non-medical influences upon medical decision-making and referral behavior: an annotated bibliography. Westport, CT: Praeger, 2003.

15. McKinlay JB, Potter DA, Feldman HA. Non-medical influences on medical decision-making. Soc Sci Med 1996;42:769-76.

16. Hajjaj FM, Salek MS, Basra MKA, et al. Non-clinical influences on clinical decision-making: a major challenge to evidence-based practice. J R Soc Med 2010;103:178-87.

17. Brown DL, Barsan WG, Lisabeth LD, et al. Survey of emergency physicians about recombinant tissue plasminogen activator for acute ischemic stroke. Ann Emerg Med 2005;46:56-60.

18. Hancock HC, Mason JM, Murphy JJ. Using the method of judgement analysis to address variations in diagnostic decision making. BMC Res Notes 2012;5:139.

19. Paunonen SV, Jackson DN. The Jackson Personality Inventory and the five-factor model of personality. J Res Pers 1996; 30:42-59.

20. Flynn D, Ford GA, Stobbart L, et al. A review of decision support, risk communication and patient information tools for thrombolytic treatment in acute stroke: lessons for tool developers. BMC Health Serv Res 2013;13:225.

21. Ryan M, Gerard K. Using discrete choice experiments to value health care programmes: current practice and future research reflections. Appl Health Econ Health Policy 2003; 2:55-64.

22. Louviere JJ, Lancsar E. Choice experiments in health: the good, the bad, the ugly and toward a brighter future. Health Econ Policy Law 2009;4:527-46.

23. Ryan M, Gerard K, Amaya-Amaya M. Using discrete choice experiments to value health and health care. Dordrecht: Springer, 2008.

24. de Bekker-Grob EW, Ryan M, Gerard K. Discrete choice experiments in health economics: a review of the literature. Health Econ 2012;21:145-72.

25. Knox MSA, Viney RC, Street DJ, et al. What's good and bad about contraceptive products? Pharmacoeconomics 2012; 30(12):1187-202

26. King MT, Hall J, Lancsar E, et al. Patient preferences for managing asthma: results from a discrete choice experiment. Health Econ 2007;16:703-17.

27. Laver K, Rehab M, Ratcliffe J, et al. Early rehabilitation management after stroke: what do stroke patients prefer? J Rehabil Med 2011;43:354-8

28. van Helvoort-Postulart D, van der Weijden T, Dellaert BGC, et al Investigating the complementary value of discrete choice experiments for the evaluation of barriers and facilitators in implementation research: a questionnaire survey. Implement Sci 2009;4:10

29. Pearson SD, Goldman L, Garcia TB, et al. Triage decisions for emergency department patients with chest pain. J Gen Intern Med 1995;10:557-64.

30. Lancsar E, Louviere J. Conducting discrete choice experiments to inform healthcare decision making. Pharmacoeconomics 2008;26:661-77.

31. Ford GA, Rodgers H, Thomson RG, et al. Development and evaluation of hyperacute services for patients with acute stroke: NIHR Programme Grant, 2007-2012.

32. Mackintosh JE, Burgess-Watson DL, Lie MLS, et al. Patient perspectives on decision making about thrombolysis in the hyperacute stroke setting. 5th International Shared Decision Making Conference; 2009, Boston, USA.

33. Murtagh MJ, Burgess-Watson DL, Jenkings KN, et al Situationally-sensitive knowledge translation and relational decision making in hyperacute stroke: a qualitative study. PLOS ONE 2012;7: e37066.

34. NVivo qualitative data analysis software; Version 9 [program], 2010.

35. Pope C, Ziebland S, Mays N. Qualitative research in health care: analysing qualitative data. BMJ 2000;320:114. 
36. Ryan M. Discrete choice experiments in health care: NICE should consider using them for patient centred evaluations of technologies. BMJ 2004;328:360.

37. Safe Implementation of Treatment-International Stroke Thrombolysis Registry (SITS-ISTR). 2013. https://sitsinternational.org/

38. Ericsson KA, Simon HA. Verbal reports as data. Psychol Rev 1980;87:215.

39. Louviere JJ, Hensher DA, Swait JD. Stated choice methods: analysis and applications. Cambridge University Press, 2000.

40. British Association of Stroke Physicians. http://www.basp.ac.uk/

41. Lancsar E, Louviere J, Flynn T. Several methods to investigate relative attribute impact in stated preference experiments. Soc Sci Med 2007;64:1738-53.
42. NHS Stroke Improvement Programme. http://www.improvement.nhs. uk/stroke/

43. Joint Royal Colleges of Physicians Training Board. Stroke Medicine (Sub Specialty). http://www.jrcptb.org.uk/trainingandcert/st3-spr/ pages/stroke-medicine.aspx

44. Intercollegiate Stroke Working Party. National clinical guideline for stroke. Royal College of Physicians of London, 2012.

45. Flynn D, Nesbitt D, McMeekin P, et al. Development and testing of a computerised decision support and risk communication tool for thrombolytic treatment in acute stroke care. 6th International Shared Decision Making Conference: Bridging the Gap Between Research and Practice: Patient Pull or Clinician Push? 2011, Maastrict, The Netherlands. 